\title{
Diamond dosimeter for small beam stereotactic radiotherapy
}

\author{
F. Marsolat, D. Tromson, N. Tranchant, M. Pomorski \\ CEA-LIST, Diamond Sensor Laboratory, Gif-sur-Yvette, France \\ D. Lazaro-Ponthus \\ CEA-LIST,LM2S, Gif-sur-Yvette, France \\ C. Bassinet, C. Huet, S. Derreumaux \\ IRSN, PRP-HOM/SDE/LDRI, Fontenay-aux-Roses, France
}

M. Chea, G. Boisserie

Pitié Salpêtrière Hospital, Paris, France

J. Alvarez

Laboratoire de Génie Electrique de Paris, CNRS UMR 8507, Gif-sur-Yvette, France

P. Bergonzo

CEA-LIST, Diamond Sensor Laboratory, Gif-sur-Yvette, France

\begin{abstract}
Recent developments of new therapy techniques using small photon beams, such as stereotactic radiotherapy, require new detectors to precisely determine the delivered dose. The dosimeter has to be as close as possible to tissue equivalence and exhibit a small detection volume compared to the size of the irradiation field, because of the lack of lateral electronic equilibrium in small beam. Characteristics of single crystaldiamond (tissue equivalent material $\mathrm{Z}=6$, high density) make it an ideal candidate to fulfill most of small beam dosimetry requirements. A commercially Element Six electronic grade synthetic diamond was selected to develop a single crystal diamond dosimeter (SCDDo) with a small detection volume $(0.4 \mathrm{~mm} 3)$. The Monte-Carlo code PENELOPE (parallelized version) was used to optimize both geometry and packaging materials of the diamond detector in order to respect the tissue-equivalent requirement. SCDDo dose profile and output factor (OF) measurements, were performed for small photon beams with a micro multileaf collimator $\mathrm{m} 3$ (BrainLab) attached to a Varian Clinac $2100 \mathrm{C}$ linear accelerator. These measurements were compared to different detectors, both active and passive: silicon diode (PTW 60017), EBT2 radiochromic films, PinPoint ionization chamber (PTW 31014). SCDDo presents an excellent spatial resolution for dose profile measurements, due its small detection volume. OFs obtained with SCDDo are very satisfactory from $0.6 \times 0.6 \mathrm{~cm} 2$ to $10 \times 10 \mathrm{~cm} 2$ field sizes, compared to PinPoint ionization chamber which underestimates OF values in small beam.
\end{abstract}

\section{Keywords}

Diamond dosimeter, Stereotactic, Small beam, Monte Carlo, Output factors 


\section{Introduction}

Radiotherapy is one of the most powerful techniques used in cancer treatment. Very specific techniques with a specific clinical objective are now used to spare the healthy tissue while tumors are irradiated. The development of stereotactic treatment has led to an increasing use of small X-ray beams, in the range of 5 to $40 \mathrm{~mm}$ in diameter. This advanced technique is used for the treatment of small tumors (less than $20 \mathrm{~cm}^{3}$ ), benign and malignant, intra- and extracranial. In Stereotactic Radiosurgery a relatively high dose is delivered in a single fraction (for instance, 90 Gy can be delivered to a patient with trigeminal neuralgia [1], [2]); in Stereotactic Radiotherapy multiple fractions of lower dose (1.8 Gy-4 Gy) are used [3]. Because of the complicated beam ballistic and realization use, the Stereotactic technique presents critical risks and requires a high accuracy in patient positioning and also in dose delivery. The accuracy in patient positioning is improved by the development of advanced imaging modalities and by fixing patient to stereotactic frame [4], [5]. Dosimetry of small beams is not very well controlled, the main issue being the determination of Output Factors (OFs). Several authors compared different commercially available detectors and Monte Carlo simulations in small beams [3], [6], [7], [8], [9]. These studies showed the large differences between OFs measured with ionization chambers, silicon diodes, films, thermo-luminescent detectors (TLD) and natural diamonds in fields smaller than $3 \mathrm{~cm} \times 3 \mathrm{~cm}$. The large active volume of detectors, their non-tissue equivalence, and the lack of lateral electronic equilibrium are the main causes of these broad results.

Recently diamond has been quoted in several papers as a good candidate as a small beam dosimeter [7], [10], [11], [12], [13]. Diamond is nearly tissue-equivalent because of its atomic number $(\mathrm{Z}=6)$ close to human tissue effective atomic number ( $Z$ eff $\sim 7.42)$. A small active volume of diamond detector allows a high spatial resolution of dose measurement, the high density of atoms in lattice $\left(10^{23}\right.$ atoms $\left./ \mathrm{cm}^{3}\right)$ keeps a high signal-to-noise ratio and diamond electric properties permit to achieve fast detector response. Many authors have studied natural diamond dosimeter commercialized by PTW [14], [15]. The non-reproducibility between devices, the high cost and the long delivery times are the main drawbacks for these detectors. Synthetic diamond is a good alternative because reproducible and optimized growth conditions permit to obtain diamond with good electronic properties and to avoid impurity incorporation. The performances of synthetic single crystal CVD for X-ray detectors were presented by various authors [11], [13], [16], [17], [18]. In our study, we focused on the design of the detector based on a commercially available single crystal CVD diamond (Element Six Ltd.) with good electronic properties as discussed later. In the present paper, Monte Carlo simulations were performed to optimize both geometry and packaging materials of the diamond detector in order to respect the small detection volume and waterequivalent requirements. The developed Single Crystal Diamond Dosimeter (SCDDo) was tested in a clinical environment with small photons beams and compared to commercially available detectors. 


\section{Optimization of single crystal diamond dosimeter using Monte Carlo simulations}

For accurate small beam dosimetry, an important requirement for the dosimeter is the waterequivalence of the device, in order to minimize the electron fluence perturbation and the energy dependence of the detector. Even if diamond is a good candidate due to its low atomic number, the packaging development remains one of the key issues to obtain small devices that match small beam dosimetry requirements. Monte Carlo (MC) simulations were performed in order to optimize the materials surrounding the diamond (electrodes, contacts bonding, encapsulation material, etc.) and to obtain a completely water-equivalent device.

\subsection{Small photon beam spectrum}

MC simulations were performed using the PENELOPE code (2006 parallelized version) developed by Salvat et al. [19]. In order to optimize the diamond detector for use in small photon beams, an energy spectrum representative of such beams is needed. This energy spectrum was obtained by simulating a $2 \times 2 \mathrm{~cm}^{2}$ field using a $\mathrm{MC}$ model of the Saturne 43 linac previously validated against experiments for a $10 \times 10 \mathrm{~cm}^{2}$ field size [20]. The Saturne 43 medical linear accelerator head geometry was modeled using PENGEOM (PENELOPE geometry code). The transport of radiation was simulated through the accelerator head. A Phase Space File (PSF) was then recorded at $90 \mathrm{~cm}$ from the source, in air, for a $6 \mathrm{MV}$ photon beam and a $2 \mathrm{~cm} \times 2 \mathrm{~cm}$ field size. This file contained data related to every particle crossing a surface of $30 \mathrm{~cm} \times 30 \mathrm{~cm}$, orthogonal to the beam axis: kind of particles, energy, position, direction, etc. This PSF was then used to study the influence of each material present in the diamond detector on the absorbed dose.

The energy photon spectra of the PSFs obtained for a $2 \times 2 \mathrm{~cm}^{2}$ and a $10 \times 10 \mathrm{~cm}^{2}$ field were compared (Fig. 1) and the main characteristic expected for a small beam was verified: the mean energy $E_{m}$ of photons increases due to the lower quantity of scattered photons in the jaws $\left(\mathrm{E}_{\mathrm{m}}=1.981 \mathrm{MeV}\right.$ for the $2 \times 2 \mathrm{~cm}^{2}$ field and $\mathrm{E}_{\mathrm{m}}=1.819 \mathrm{MeV}$ for the $10 \times 10 \mathrm{~cm}^{2}$ field). This result allows us to verify that the $2 \times 2 \mathrm{~cm}^{2}$ photon beam spectrum simulated is representative of a small beam and can be used to optimize the geometry and materials of SCDDo.

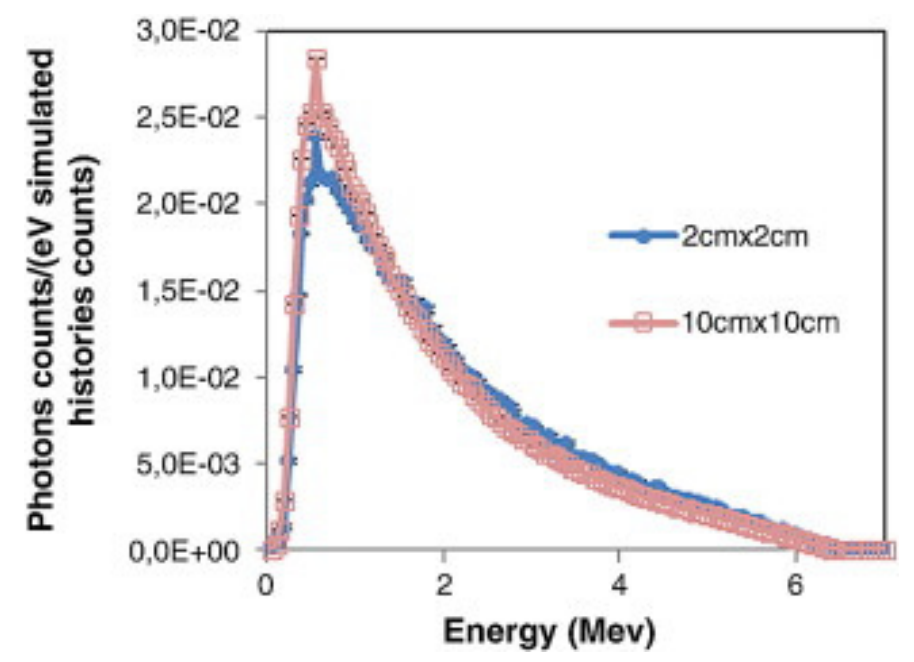

Fig. 1. Energy spectra of photons calculated in air, at $90 \mathrm{~cm}$ from the target of a Saturne 43 linac, for $6 \mathrm{MV}$ photon beams, for a $2 \times 2 \mathrm{~cm}^{2}$ and a $10 \times 10 \mathrm{~cm}^{2}$ field. Spectra resolution: $50 \mathrm{keV}$. Spectra are normalized to the total number of photons recorded in each PSF. 


\subsection{Modelling of SCDDo}

Diamond was modeled as a carbon material, with a density of $3.51 \mathrm{~g} / \mathrm{cm}^{3}$ and mean excitation energy of $87.6 \mathrm{eV}$ [21]. Single-crystal diamond dimensions modeled in this work were $3 \mathrm{~mm} \times 3 \mathrm{~mm}$ with a thickness varying from $100 \mu \mathrm{m}$ to $500 \mu \mathrm{m}$. Circular metallic electrodes were simulated as two cylinders of $1 \mathrm{~mm}$ in diameter, located on both sides of the diamond in a sandwich configuration. These electrodes delimit the sensitive volume of SCDDo (Fig. 2a). A $1 \mathrm{~mm}$ electrode diameter was a good compromise between high signalto-noise ratio and small detection volume required for a high resolution detector. Different materials were simulated for the electrodes: gold and nickel electrodes respectively on each diamond face [10], and aluminum electrodes on both sides. Different electrode thicknesses were simulated (from $0.1 \mu \mathrm{m}$ to $10 \mu \mathrm{m}$ ) to optimize their thicknesses in order to avoid perturbation of the absorbed dose in the diamond detector. The volume of the conductive glue used to connect conductive wires $(100 \mu \mathrm{m}$ in diameter) to electrodes was not negligible and was also modeled in PENGEOM as cylinders of $1 \mathrm{~mm}$ in diameter with a thickness of $250 \mu \mathrm{m}$, in graphite or silver. In practice, glue contact dimensions were not so large, but the worst situation was simulated. The second extremity of thin wires connected to electrodes was bonded to a triaxial cable. The geometry of the triaxial cable was modeled according to technical data sheet, in order to determine the minimal distance $d$ between diamond and cable necessary to reduce perturbation of the dose deposited in the diamond (Fig. 2b). Diamond electrodes and electrical connections have to be encapsulated in water equivalent material to protect the detector. Three different polymers were modeled: polydimethylsiloxane (PDMS), polymethylmethacrylate (PMMA) and polybenzylemethacrylate (PBnMA). Their composition and density make them of interest for water equivalent encapsulation (Table 1). Encapsulation material was simulated as a cylinder of $5.6 \mathrm{~mm}$ in diameter and $5 \mathrm{~mm}$ in height (Fig. 2c). For all MC simulations performed in this work, the center of diamond was positioned on the beam axis, at $5 \mathrm{~cm}$ depth in a $20 \mathrm{~cm} \times 20 \mathrm{~cm} \times 20 \mathrm{~cm}$ water tank. The source-detector distance was $100 \mathrm{~cm}$.

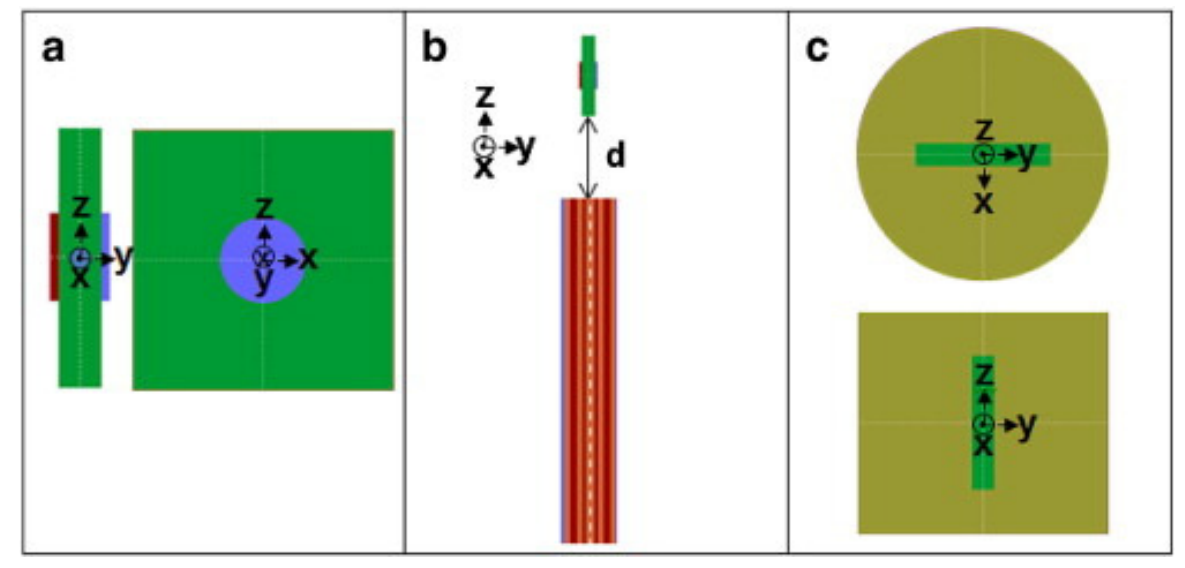

Fig. 2. Different geometries of diamond detector modeled using PENGEOM. a: Diamond of $3 \mathrm{~mm} \times 3 \mathrm{~mm} \times 0.5 \mathrm{~mm}$, with electrodes of $1 \mathrm{~mm}$ in diameter and $0.25 \mathrm{~mm}$ thick in a sandwich configuration: study of the influence of the electrodes on absorbed dose to diamond. b: Study of the influence of the triaxial cable on absorbed dose to diamond as a function of distance $d$ between the diamond and the cable. c: Diamond of $3 \mathrm{~mm} \times 3 \mathrm{~mm} \times 0.5 \mathrm{~mm}$ encapsulated in a polymer material cylinder (5.6 $\mathrm{mm}$ in diameter, $5 \mathrm{~mm}$ in height): study of the influence of encapsulation material on absorbed dose to diamond. 
Table 1. Chemical formula and density of different encapsulation materials for small beam diamond dosimeter.

\begin{tabular}{|l|l|l|l|}
\hline Encapsulation material & PDMS & PMMA & PBnMA \\
\hline Chemical formula & $\left(\mathrm{C}_{2} \mathrm{H}_{6} \mathrm{OSi}\right)_{\mathrm{n}}$ & $\left(\mathrm{C}_{5} \mathrm{H}_{8} \mathrm{O}_{2}\right)_{\mathrm{n}}$ & $\left(\mathrm{C}_{11} \mathrm{H}_{12} \mathrm{O}_{2}\right)_{\mathrm{n}}$ \\
\hline Density & 0.96 & 1.19 & 1.18 \\
\hline
\end{tabular}

\subsection{MC simulations parameters}

To achieve low relative uncertainty in small volumes like electrodes $(<1 \%)$, a high number of simulated particles and hence long calculation time were needed. The values of some PENELOPE user-defined parameters were carefully chosen in order to speed up simulations and preserve a high accuracy. EABS is the cutoff energy defined for each kind of particles: electrons, positrons and photons. The transport of a particle is discontinued when its energy reaches the EABS value: the particle is then assumed to be absorbed in the medium and its energy is locally deposited. Four other parameters are specific to electron and positron transport: C1, C2, WCC and WCR. C1 and C2 define the average angular deflection and the maximum average fractional energy loss between consecutive hard elastic events, respectively. $\mathrm{C} 1$ and $\mathrm{C} 2$ can take values from 0 to 0.2 . If both parameters are set to zero, the simulation is performed in a very detailed way but takes a long time. The simulation is speeded up using larger values of $\mathrm{C} 1$ and $\mathrm{C} 2$, at the expense of accuracy. WCC and WCR are the cutoff energy loss for hard inelastic collisions and hard bremsstrahlung emission, respectively. To achieve a good energy resolution, WCC and WCR should have small values. These parameters were selected in order to have a high accuracy in diamond detector, but it implies long calculation time: EABS was set to $0.5 \mathrm{keV}$ for all kind of particles, $\mathrm{C} 1$ and $\mathrm{C} 2$ were set to 0.01 , WCR and WCC were equal to $0.05 \mathrm{eV}$. To speed up simulations, these parameter values were successively increased in regions surrounding the diamond detector, where lower accuracy was needed, as described by Gorka et al. [21] (Table 2).

Table 2. PENELOPE parameters selected to study the influence of electrodes on the absorbed dose to diamond. These parameters determine the accuracy and the speed of the simulation.

\begin{tabular}{|l|l|l|l|l|l|}
\hline \multirow{2}{*}{ Region } & \multicolumn{2}{l|}{ EABS (keV) } & \multirow{2}{*}{ C1, C2 } & WCC (keV) & WCR (keV) \\
\cline { 2 - 5 } & $\begin{array}{l}\text { Electrons, } \\
\text { positrons }\end{array}$ & Photons & & & \\
\hline 1 (Diamond and electrodes) & 0.5 & 0.5 & 0.01 & $0.05 \times 10^{-3}$ & $0.05 \times 10^{-3}$ \\
\hline 2 (Region 1 $+0.1 \mathrm{~cm}$ of water) & 1 & 1 & 0.05 & 1 & 1 \\
\hline 3 (Region 2 +1 cm of water) & 100 & 5 & 0.1 & 10 & 10 \\
\hline 4 (Remaining water) & 500 & 5 & 0.2 & 10 & 10 \\
\hline
\end{tabular}




\subsection{Influence of detector materials surrounding the diamond on the absorbed dose}

To study the influence of electrodes on the absorbed dose to the diamond, the ratio $\mathrm{D} / \mathrm{D}_{0}$ was calculated using $\mathrm{MC}$ simulations, $\mathrm{D}$ being the mean absorbed dose to the sensitive volume of the diamond in presence of electrodes and $\mathrm{D}_{0}$ being the mean absorbed dose in absence of electrodes. To obtain a water-equivalent device, this ratio had to be close to 1 . Results are displayed in Fig. 3 as a function of diamond thickness, electrode material and thickness. For gold-nickel electrodes, the mean absorbed dose to diamond is increased up to $3.3 \%$ because of the increase of electrons scattering in high- $\mathrm{Z}$ material $(\mathrm{Z}$ gold $=79, \mathrm{Z}$ nickel $=28)$. This dose enhancement is emphasized when electrode thickness is increased. Electrons passing through high-Z electrodes are more scattered in various directions than low-Z materials. As high Zelectrodes are used, electron fluence in the diamond and the energy deposited in the diamond increase. As expected, low- $Z$ aluminum electrodes have no influence on the absorbed dose to the diamond for the thickness range of 0.1 to $10 \mu \mathrm{m}$ in small photon beams.
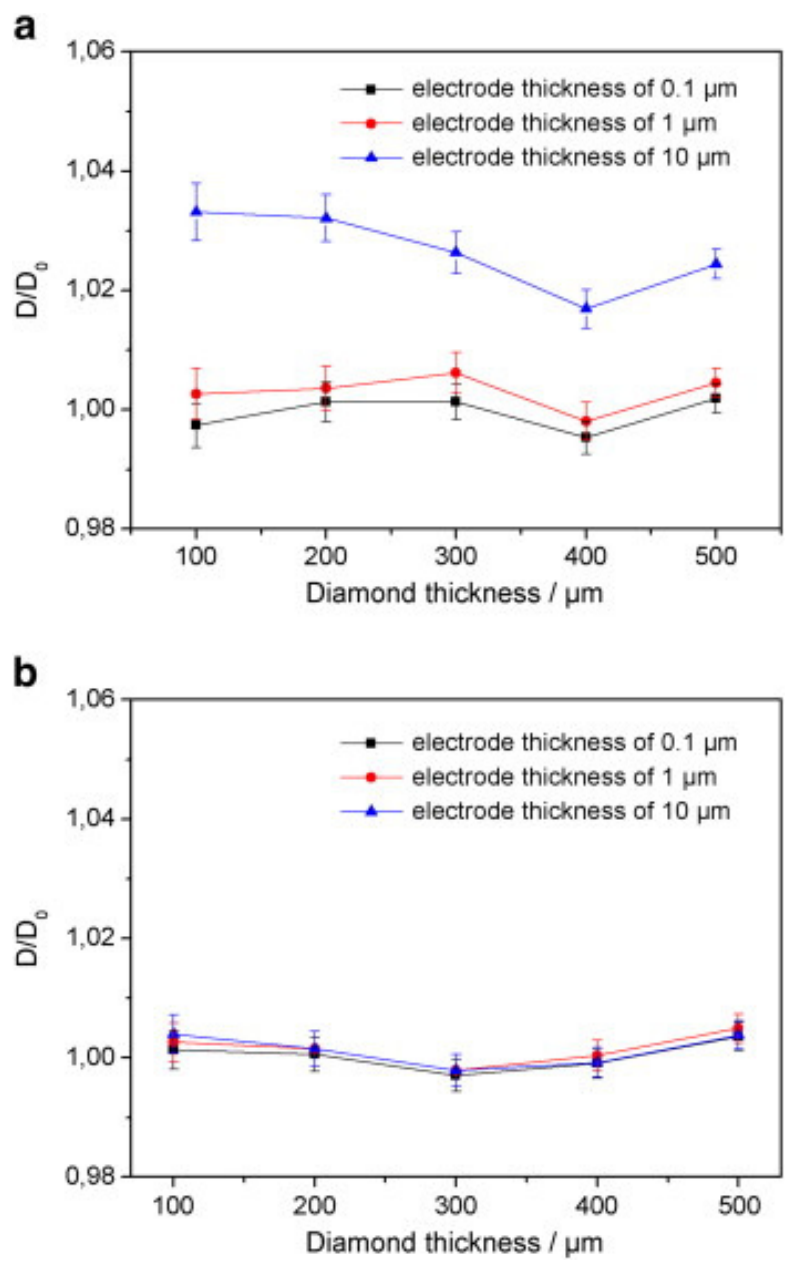

Fig. 3. Study of the influence of $1 \mathrm{~mm}$ diameter electrodes on the absorbed dose in the diamond in regard to the ratio of the mean absorbed dose $\mathrm{D}$ in the diamond sensitive volume in presence of electrodes to the mean absorbed dose $\mathrm{D}_{0}$ without electrodes, for a $6 \mathrm{MV}$ photon beam and a $2 \mathrm{~cm} \times 2 \mathrm{~cm}$ field size. a: Results for gold electrode on one diamond face and nickel electrode on the second face. $\mathrm{b}$ : Results for aluminum electrodes on both sides of the diamond. 
Fluence perturbation in the diamond was higher with silver conductive glue used for contacting wires to electrodes than with carbon conductive glue. Actually, silver artificially increases the effective electrode thickness. For diamond thickness of $500 \mu \mathrm{m}$ (Element Six diamond sample thickness) and aluminum electrode thickness of $0.1 \mu \mathrm{m}, \mathrm{D} / \mathrm{D}_{0}$ was $1.115 \pm 0.43 \%$, D being the mean absorbed dose to diamond in presence of silver glue and $\mathrm{D}_{0}$ the absorbed dose to diamond without silver. When silver glue was replaced with conductive graphite glue, $\mathrm{D} / \mathrm{D}_{0}$ was $0.996 \pm 0.41 \%$, due to the low-Z of carbon. Some authors use high-Z material contacts in their diamond dosimeter [12], [13] but the presence of non-water-equivalent materials close to the diamond involves electron fluence perturbations in the sensitive volume of the diamond and an energy dependence of the detector response.

The triaxial cable has no influence on the absorbed dose to diamond in a small beam of $2 \times 2 \mathrm{~cm}^{2}$ when it is positioned parallel to the beam axis at a minimum distance of $1 \mathrm{~cm}$ from the diamond (Table 3 ). For a distance $d=0 \mathrm{~cm}$, the increase of the mean absorbed dose to the diamond was caused by the fluence of electrons backscattered from copper present in cable.

Table 3. Calculated $\mathrm{D} / \mathrm{D}_{0}$ as a function of the distance $\mathrm{d}$ between the diamond and the triaxial cable. $\mathrm{D}$ is the mean absorbed dose to diamond in presence of the triaxial cable and $\mathrm{D}_{0}$ is the mean absorbed dose to diamond in absence of the triaxial cable, for a $6 \mathrm{MV}$ photon beam and a $2 \times 2 \mathrm{~cm}^{2}$ field size.

\begin{tabular}{|c|l|}
\hline Distance d between diamond and triaxial cable $(\mathrm{cm})$ & $\mathrm{D} / \mathrm{D}_{0}$ \\
\hline 0 & $\begin{array}{l}1.023 \\
( \pm 0.37 \%)\end{array}$ \\
\hline 1 & $\begin{array}{l}1.001 \\
( \pm 0.37 \%)\end{array}$ \\
\hline 2 & $\begin{array}{l}1.000 \\
( \pm 0.37 \%)\end{array}$ \\
\hline 3 & $\begin{array}{l}1.000 \\
( \pm 0.37 \%)\end{array}$ \\
\hline
\end{tabular}

Finally, MC simulations of encapsulation material showed that PDMS was not sufficiently water-equivalent, because the mean absorbed dose to diamond was increased in presence of this surrounding material: $\mathrm{D} / \mathrm{D}_{0}=1.020 \pm 0.32 \%$, with $\mathrm{D}$ the absorbed dose to diamond in presence of PDMS. Hence, PMMA and PBnMA were shown to be optimal materials for diamond detector encapsulation, with respectively $\mathrm{D} / \mathrm{D}_{0}$ of $0.997 \pm 0.32 \%$ and $0.993 \pm 0.33 \%$.

\subsection{Water-equivalent SCDDo}

Water-equivalent small beam dosimeter was developed with single crystal diamond, using the results of MC simulations (Fig. 4). The sensitive volume was delimited by aluminum electrodes of $1 \mathrm{~mm}$ in diameter and $100 \mathrm{~nm}$ in thickness. Electrodes were deposited on both sides of the diamond, by photolithography process and evaporation system. Materials present in this device were optimized in order to respect the low- $Z$ requirements for small beam 
dosimetry and obtain almost a water-equivalent detector: aluminum electrodes, aluminum wires of $100 \mu \mathrm{m}$ in diameter, conductive graphite glue, polybenzylmethacrylate (PBnMA) and polymethylmethacrylate (PMMA) encapsulation. The triaxial cable was connected at a distance larger than $1 \mathrm{~cm}$ in order to avoid perturbation of the deposited dose in the diamond. Finally, conductive colloid graphite covered the device and was connected to ground in order to reduce the noise from external signals. The position of detection volume in the water-equivalent housing was verified with X-ray radiography.

a

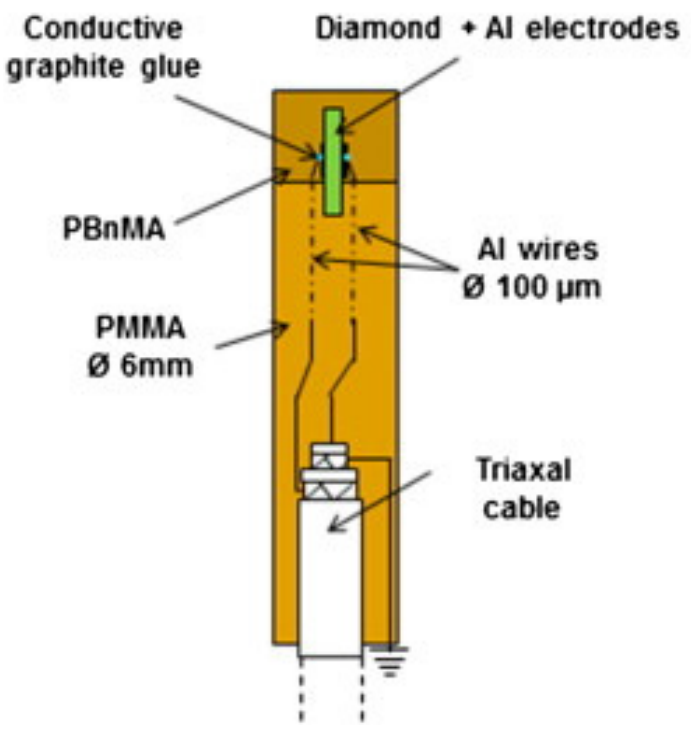

b

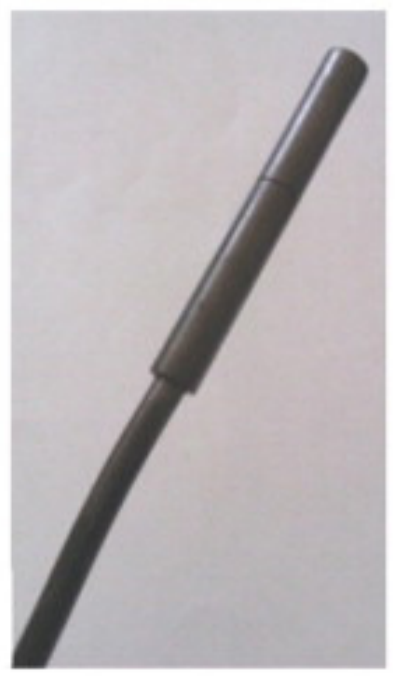

C

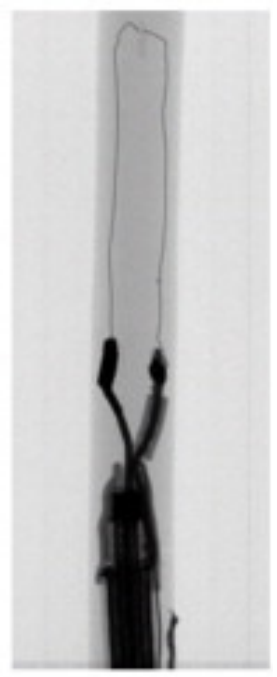

Fig. 4. a. Schema of water-equivalent SCDDo.

b. Photography of diamond detector covered with conductive graphite.

c. X-ray radiography of the device.

\section{Measurements with single crystal diamond dosimeter}

\subsection{Characterization of single crystal diamond sample}

To develop a Single Crystal Diamond Dosimeter, we selected one Element Six electronic grade synthetic sample with good crystalline quality and good electronic properties as described further. The sample size is $2 \mathrm{~mm} \times 2 \mathrm{~mm} \times 500 \mu \mathrm{m}$. Crossed polarizers and Scanning Electron Microscopy (SEM) images are shown in Fig. 5. 


\section{a}

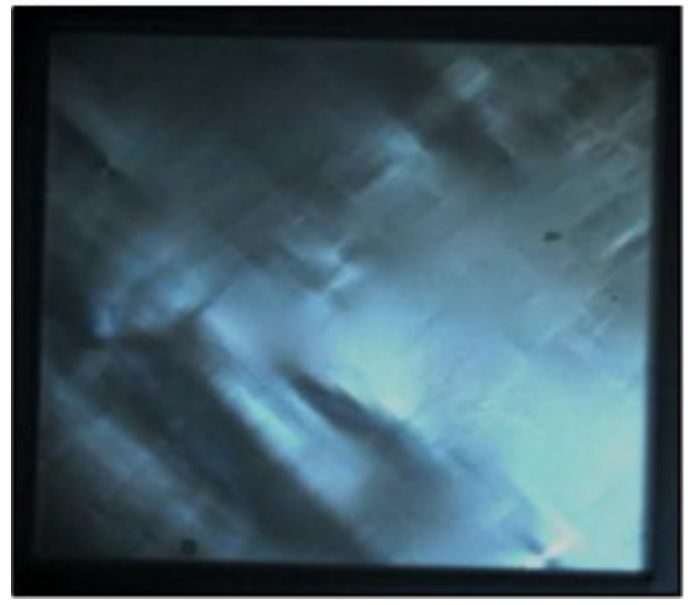

b

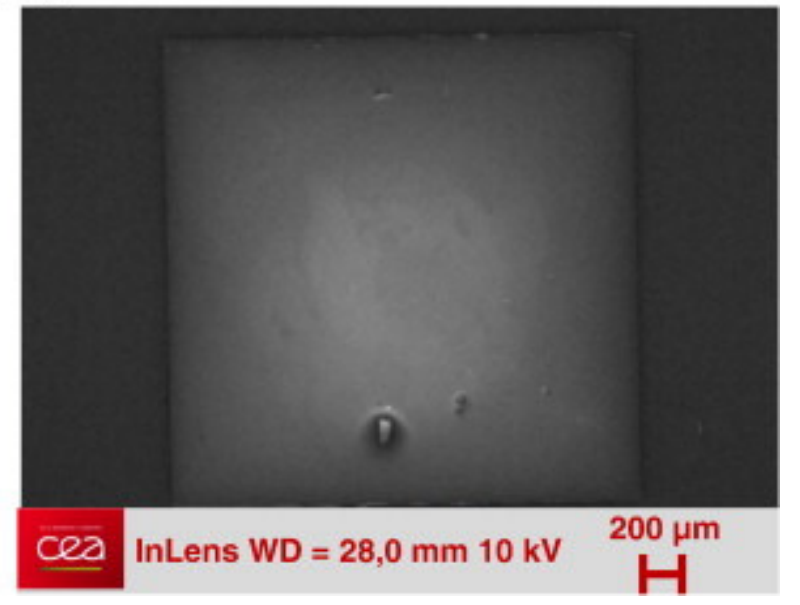

Fig. 5. Birefringence (a) and SEM images (b) of Element Six synthetic single crystaldiamond selected for diamond dosimeter.

E6 diamond sample crystallographic quality (stress, defects, etc.) were checked using confocal Raman spectroscopy, with a $532 \mathrm{~nm}$ excitation from a WITec spectrometer. A surface of $50 \mu \mathrm{m} \times 50 \mu \mathrm{m}$ of this single crystal diamond was scanned with a laser spot of $5 \mu \mathrm{m}$ in diameter. Fig. 6a shows the homogeneity of the surface. The average Raman spectrum calculated on this surface presents a single sharp peak at $1332.27 \mathrm{~cm}^{-1} \pm 0.14 \mathrm{~cm}^{-1}$ with a Full-Width at Half-Maximum (FWHM) of $3.34 \mathrm{~cm}^{-1} \pm 0.05 \mathrm{~cm}^{-1}$, indicating a good crystalline quality (Fig. 6b) [22], [23], [24].

a

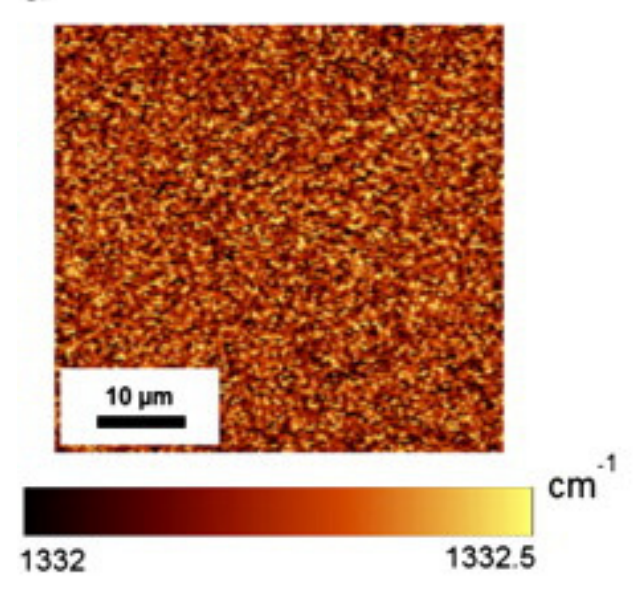

b

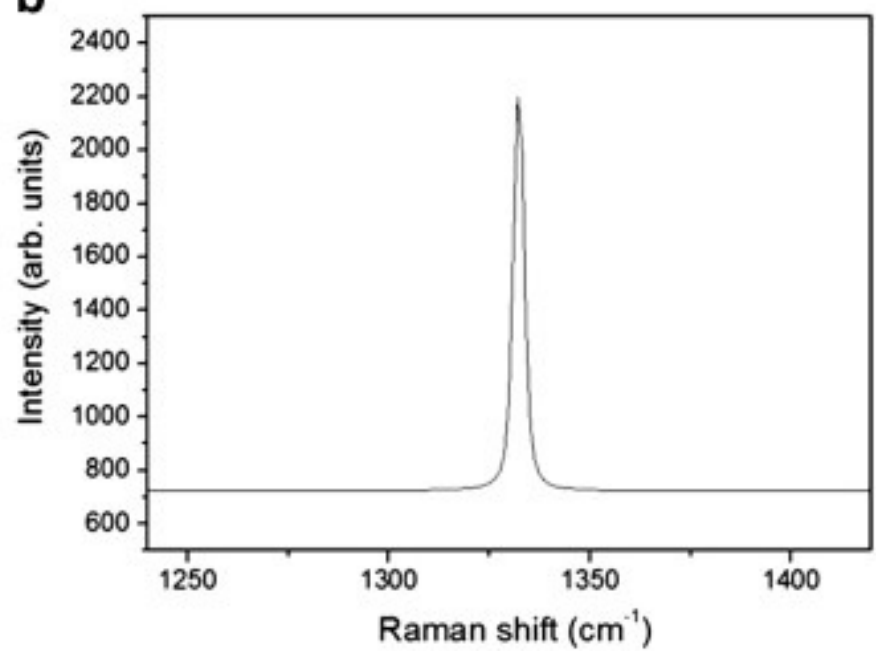

Fig. 6. a: Diamond peak position measured with confocal micro-Raman spectroscopy on a surface of $50 \mu \mathrm{m} \times 50 \mu \mathrm{m}$.

b: Average Raman spectrum calculated on the whole scanned surface.

Electronic characterization was performed on this sample with alpha-particle measurements and Transient Current Technique (TCT), in order to determine charge carrier drift velocity and mobility values (Fig. 7). Mobility curve fit as described in many papers [16], [25], [26], [27] yields zero-field mobility values of 
$\mu_{0, \mathrm{~h}}=(1959 \pm 37) \mathrm{cm}^{2} \mathrm{~V}^{-1} \mathrm{~s}^{-1}$ and $\mu_{0, \mathrm{e}}=(1730 \pm 51) \mathrm{cm}^{2} \mathrm{~V}^{-1} \mathrm{~s}^{-1}$,

for holes and electrons respectively, and saturation velocity values of

$\mathrm{V}_{\mathrm{s}}^{\mathrm{h}}=\left(1.29 \times 10^{7} \pm 6.1 \times 10^{5}\right) \mathrm{cm} \mathrm{s}^{-1}$ and $\mathrm{v}_{\mathrm{s}}^{\mathrm{e}}=\left(8.45 \times 10^{6} \pm 5.8 \times 10^{5}\right) \mathrm{cm} \mathrm{s}^{-1}$

for holes and electrons respectively. These values are comparable to those obtained by Pernegger et al. [26] with an Element Six single crystal diamond. Charge Collection Efficiency (CCE) was also measured with alpha-particles at various electric fields, comparatively to a $100 \%$ efficient PIN silicon diode. At electric field higher than $0.8 \mathrm{~V} / \mu \mathrm{m}$, CCE is approximately $100 \%$, indicating that all carriers are collected by the electrodes.
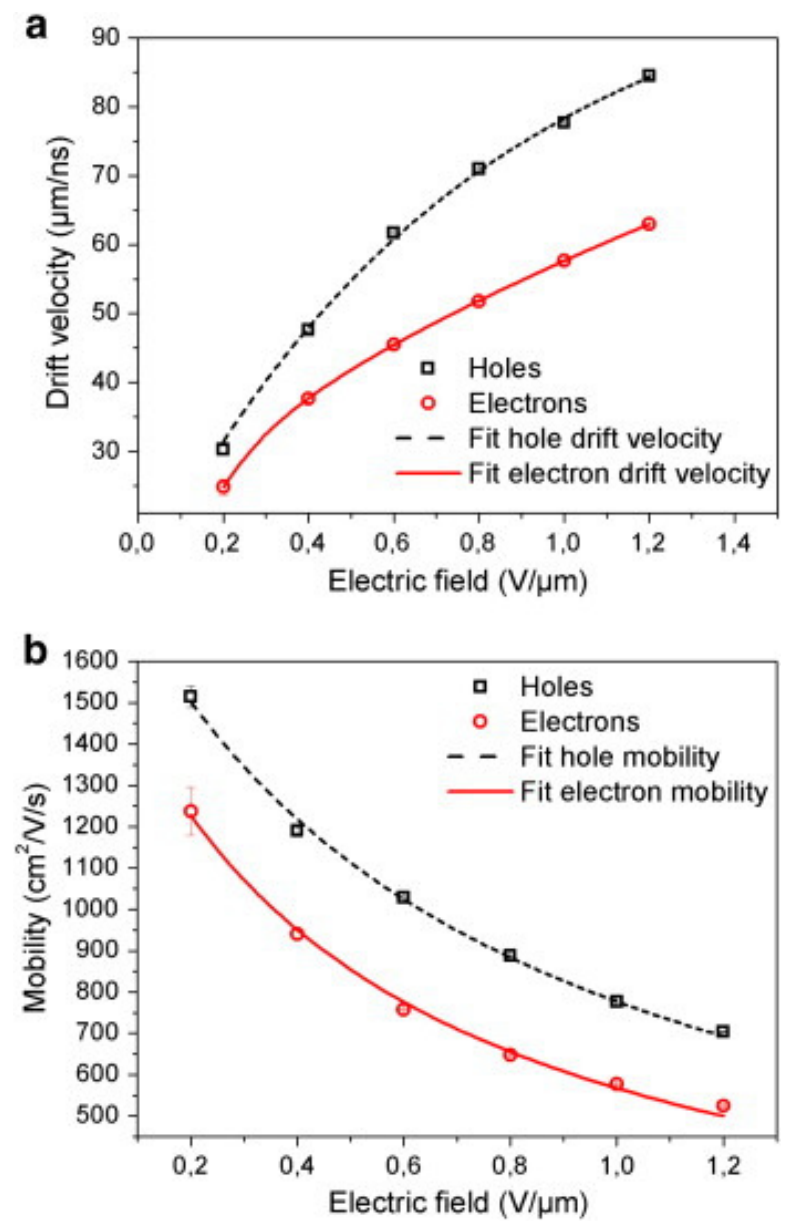

Fig. 7. Drift velocity (a) and mobility (b) values as a function of applied electric field.

The mounted detector with Element Six diamond exhibits a small detection volume of approximately $0.4 \mathrm{~mm}^{3}$, as required for small beam dosimetry. The stability of the device was evaluated with an X-ray tube $(50 \mathrm{kV}, 1 \mathrm{~mA}, 14,400 \mathrm{~Gy} / \mathrm{h}$ at the point of measurement) by determining the percentage ratio of the standard deviation to the mean current value induced by $\mathrm{X}$-rays. The stability was determined during a short irradiation time in order to estimate the value in the most unfavorable conditions. A stability of $0.9 \%$ was observed for $60 \mathrm{~s}$ of irradiation, after $5 \mathrm{~min}$ of pre-irradiation. A high signal to noise ratio of 10,000 was obtained with this detector. 


\subsection{Clinical environment measurements in small beams}

SCDDo measurements were performed at La Pitié Salpêtrière Hospital (Paris, France), with a Varian Clinac $2100 \mathrm{C}$ medical linear accelerator. A micro multileaf collimator system ( $\mu$ MLC m3, BrainLab) dedicated to stereotactic treatments was attached to this accelerator. This $\mu \mathrm{MLC}$ allows conformal shaping of the small beam in order to only irradiate small tumors. Measurements were performed in a PTW motorized water tank, with a photon beam of $6 \mathrm{MV}$ and a dose rate of $400 \mathrm{MU} / \mathrm{min}$. The diamond detector was placed at a source-surface distance (SSD) of $100 \mathrm{~cm}$ and a depth of $10 \mathrm{~cm}$ in water (ESTRO-IAEA recommendations [28]). SCDDo was positioned in the water tank with its cable parallel to the beam axis as simulated before. It was polarized at $100 \mathrm{~V}$. The priming dose was not investigated in this work but a large pre-irradiation dose of $10 \mathrm{~Gy}$ was delivered to SCDDo before performing measurements. Repeatability was studied with five consecutive irradiations with a constant dose of $300 \mathrm{MU}$; the coefficient of variation determined by the ratio of standard deviation to mean charge was $0.1 \%$. Dose linearity of SCDDo response was verified for a $10 \mathrm{~cm} \times 10 \mathrm{~cm}$ field size, by measuring the charge induced by irradiation with a dose range of 10 to $800 \mathrm{MU}$. Dose rate dependence of the detector response was evaluated by changing the pulse repetition frequency of the accelerator $(80 \mathrm{MU} / \mathrm{min}-400 \mathrm{MU} / \mathrm{min}$ range). For these measurements, SCDDo was connected to a PTW UNIDOS electrometer. Dose linearity was observed with a linearity coefficient $\mathrm{R}^{2}$ equal to 1 (Fig. 8). The SCDDo behavior with dose rate was analyzed with the Fowler's model [29] described by the following equation:

$\mathrm{I}=\mathrm{I} 0+\mathrm{R} \cdot \mathrm{D} \Delta$

where $\mathrm{I}$ is the SCDDo current, $\mathrm{I}_{0}$ the dark current and $\Delta$ the fitting parameter that describes the deviation to linearity. This last parameter has to be as close as possible to 1 to have a dose rate linearity of the detector response. Fig. 9 shows the SCDDo current as a function of dose rate and the corresponding fitting curve according to Fowler's equation. The result of the fitting gives a $\Delta$ value of $0.975 \pm 0.003$, in good agreement with those obtained with natural diamond of PTW dosimeter [15], [30] and with other synthetic single crystal diamonds [31].

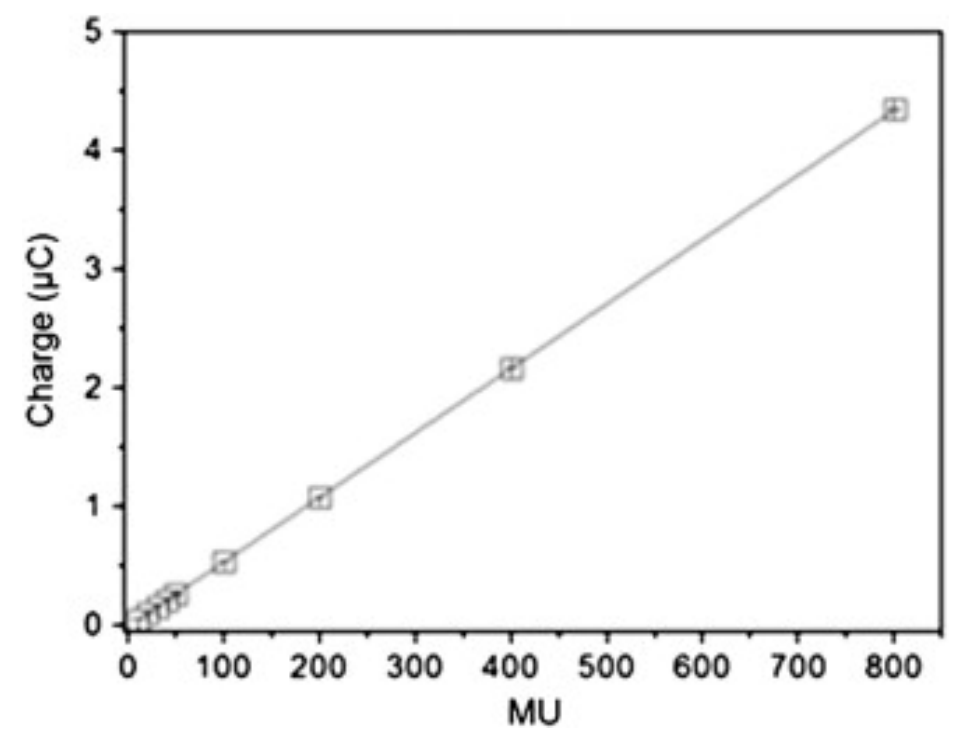

Fig. 8. Dose dependence of SCDDo response. Error bars are less than the height of data points $(\square)$. Linear fit is plotted with solid line. 


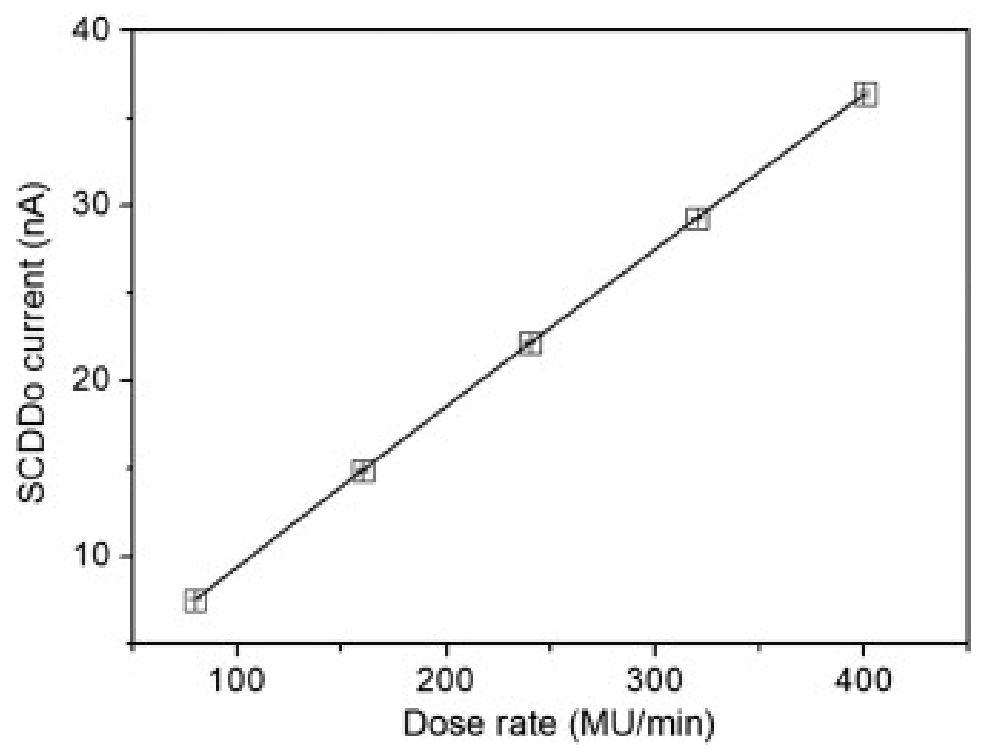

Fig. 9. Dose rate dependence of SCDDo response. Error bars are less than the height of data points ( $\square)$. Fowler's equation fit is plotted with solid line.

Cross-line dose profiles were measured with SCDDo for the smallest field size available with the $\mu$ MLC $\mathrm{m} 3\left(0.6 \times 0.6 \mathrm{~cm}^{2}\right)$, by positioning the cable parallel to the beam axis and the smallest dimension of the diamond detection volume (its thickness of $500 \mu \mathrm{m}$ ) in the scanning direction, in order to have the best spatial resolution available. These dose profiles were compared to two commercially available detectors providing a good spatial resolution: silicon diode (PTW 60017) and EBT2 Gafchromic film [6], [7], [32], [33]. This diode is a ptype silicon diode, operated at $0 \mathrm{~V}$, with a disk-shaped sensitive volume perpendicular to the detector axis. Its detection volume has dimensions of $0.6 \mathrm{~mm}$ in diameter and $30 \mu \mathrm{m}$ in thickness. For these measurements, the diode was also positioned with its cable parallel to the beam axis in the water tank as recommended by the constructor. A PTW Tandem Dual Channel electrometer and Mephysto software were used for dose profile measurements with the diode and SCDDo. Dose profile measurements with the EBT2 films were performed in a solid water phantom. The films were read $24 \mathrm{~h}$ after irradiation with an EPSON Dual Lens Perfection V700 scanner. The cross-line dose profiles were displayed in Fig. 10. The $20 \%-80 \%$ penumbras were calculated for these dose profiles: $1.6 \mathrm{~mm}$ for SCDDo, $1.8 \mathrm{~mm}$ for EBT2 films and silicon diode. These penumbra values confirmed the very good spatial resolution of the diamond detector, thanks to its small detection volume, with this orientation in the photon beam. Results are slightly better than those obtained with the diode and Gafchromic films, which are considered nowadays as the best spatially resolved detectors available for small beams. 


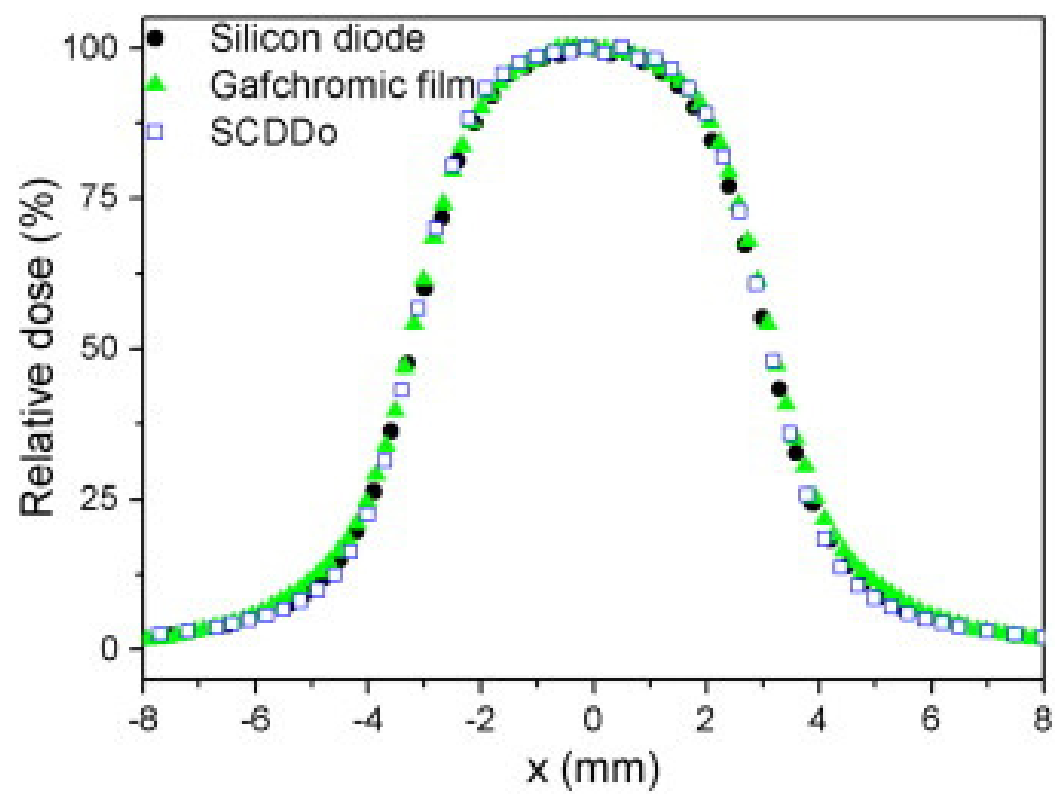

Fig. 10. Cross-line dose profiles measured with SCDDo, the PTW diode 60017 and Gafchromic films, for a $6 \mathrm{MV}$ photon beam and a $6 \times 6 \mathrm{~mm}^{2}$ beam size, with a Varian Clinac $2100 \mathrm{C}$ linac and a $\mu$ MLC m3. Depth of measurements: $10 \mathrm{~cm}$ in water. SSD of $100 \mathrm{~cm}$.

OFs measured with SCDDo were compared to those obtained with the PTW 31014 PinPoint ionization chamber, from $0.6 \mathrm{~cm} \times 0.6 \mathrm{~cm}$ to $10 \mathrm{~cm} \times 10 \mathrm{~cm}$ field sizes. Both detectors were connected to a PTW UNIDOS electrometer and they were positioned vertically with the stem and cable aligned with the beam to ensure their uniform irradiation [6]. The PTW 31014 PinPoint ionization chamber is the miniaturized ionization chamber commercially available for small beam and is known as a good reference detector for beam sizes from $3 \mathrm{~cm} \times 3 \mathrm{~cm}$ to $10 \mathrm{~cm} \times 10 \mathrm{~cm} \mathrm{[6],} \mathrm{[7],} \mathrm{[34].} \mathrm{It} \mathrm{operates} \mathrm{at} \mathrm{the} \mathrm{nominal} \mathrm{voltage} \mathrm{of} 400 \mathrm{~V}$ and exhibits a volume of $15 \mathrm{~mm}^{3}$ ( $2 \mathrm{~mm}$ diameter by $5 \mathrm{~mm}$ length). OFs results were displayed in Fig. 11. For field sizes larger than $3 \mathrm{~cm} \times 3 \mathrm{~cm}$, SCDDo and the PinPoint chamber are in good agreement. For smallest beams (less than $3 \mathrm{~cm} \times 3 \mathrm{~cm}$ ), the PTW 31014 PinPoint chamber underestimates OFs due to its too large detection volume compared to the beam size. Since the dose measured with a detector is the average dose in the whole detection volume, the dose measured on the beam axis of a small beam is underestimated with a large detection volume. Moreover, the presence of air in an ionization chamber increases the loss of lateral electronic equilibrium, decreasing the dose measured on the beam axis of a small beam. The results obtained with SCDDo are very satisfactory because the OF values are higher than those obtained with the PinPoint detector, with a maximum difference of $7 \%$ for the $6 \mathrm{~mm} \times 6 \mathrm{~mm}$ field size. 


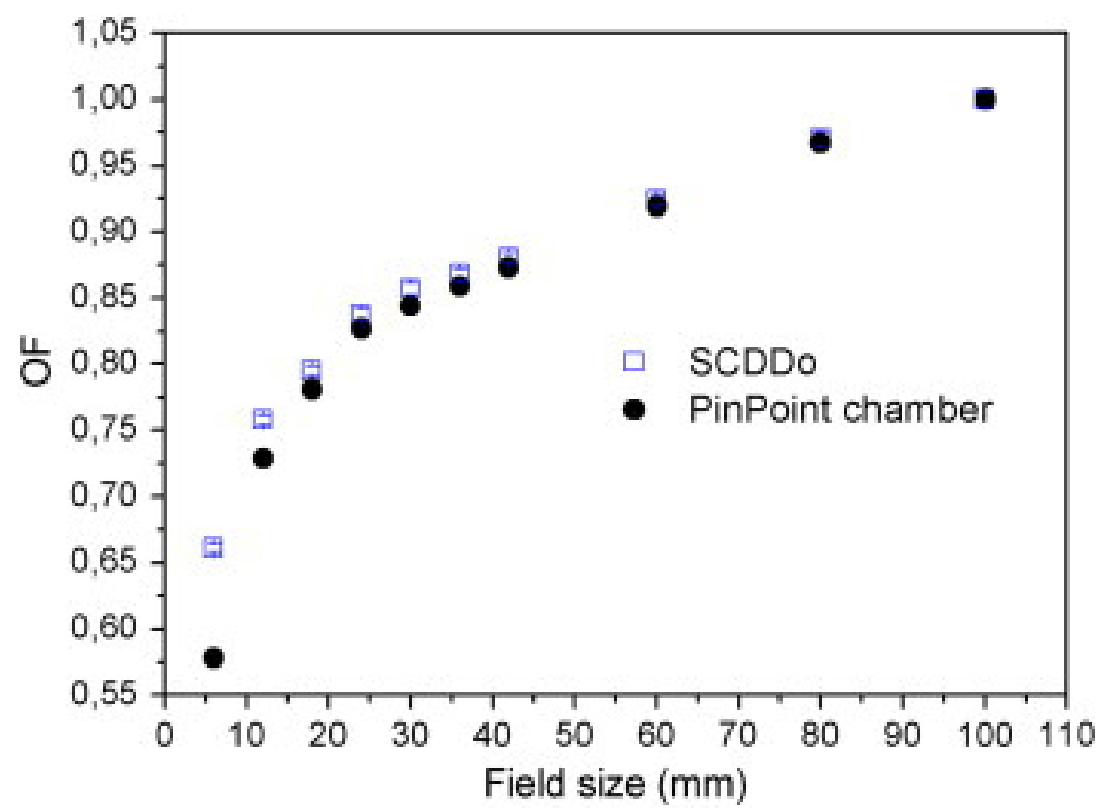

Fig. 11. Output factors measured with SCDDo (square data points and error bars) and a PinPoint chamber (circle data points), for 6MV photon beam, with a Varian Clinac $2100 \mathrm{C}$ linac and a $\mu \mathrm{MLC} \mathrm{m} 3$. Depth of measurements: $10 \mathrm{~cm}$ in water. SSD of $100 \mathrm{~cm}$.

To our knowledge, it is the first study demonstrating that a single crystal CVD diamond gives OF values higher than a PinPoint ionization chamber in a small beam. Ciancaglioni et al. [12] concluded that OF values measured with their diamond detector were similar to those obtained with a PinPoint ionization chamber, because of their large electrode dimensions ( $2.2 \mathrm{~mm}$ diameter) and the dose averaging effect. However, a future study is necessary to conclude that our device gives the exact OF values in small beam. For that, the comparison with other detectors like silicon diode and with Monte Carlo simulations will be performed in stereotactic beams.

\section{Conclusions}

The composition and the density of a dosimeter are the main factors influencing the detector response in small beams, in addition to a small active volume. Materials present in our SCDDo were determined using Monte Carlo simulations in order to obtain a complete water equivalent device and to reduce electron fluence perturbations in the sensitive volume of the diamond.

Clinical environment measurements were performed to evaluate the suitability of our waterequivalent SCDDo for stereotactic radiotherapy dosimetry. An excellent dose linearity of SCDDo response was measured with $6 \mathrm{MV}$ photon beam, in a dose range of $6.7 \mathrm{cGy}$ to $532.6 \mathrm{cGy}$ with a dose rate of $2.7 \mathrm{~Gy} / \mathrm{min}$. Dose profiles measured with the diamond for the smallest field size available with the $\mu$ MLC- $\mathrm{m} 3(6 \mathrm{~mm} \times 6 \mathrm{~mm})$ present an excellent spatial resolution due its small detection volume. The 20\%-80\% penumbras measured with the diamond are slightly smaller than those measured with the EBT2 films and a PTW 60017 diode, when SCDDo was positioned with its thin sides parallel to the scanning direction.

Finally, OFs measured with SCDDo for field sizes smaller than $3 \mathrm{~cm} \times 3 \mathrm{~cm}$ are higher than those measured with a PTW 31014 PinPoint ionization chamber which is well known to 
underestimate the OF values. In a future work, SCDDo OFs in small beams will be compared to other detectors, both active and passive (diode, films and TLD), and with Monte Carlo simulations.

\section{Prime novelty statement}

To our knowledge, it is the first study demonstrating that a water-equivalent single crystal CVD diamond dosimeter performs better than a PinPoint ionization chamber in measuring output factors in a small beam.

\section{Acknowledgments}

The authors would like to acknowledge F. Moignau and L. De Carlan from LNHB (CEA) for their contribution to this work. This work was performed in the framework of project "DIADOMI" which is granted by the French National Research Agency.

\section{References}

[1] D. Kondziolka, L.D. Lunsford, J.C. Flickinger. Stereotact. Funct. Neurosurg., 70 (1998), pp. 187-191

[2] D. Kondziolka, L.D. Lunsford, J.C. Flickinger. Clin. J. Pain, 18 (2002), pp. 42-47

[3] I.J. Das, M.B. Downes, A. Kassaee, Z. Tochner. J. Radiosurg., 3 (2000), pp. 177-186

[4] F. Baba, Y. Shibamoto, N. Tomita, C. Ikeya-Hashizume, K. Oda, S. Ayakawa, H. Ogino, C.Sugie. Radiat. Oncol., 4 (2009), p. 15

[5] J. Wulf, U. Hädinger, U. Oppitz, B. Olshausen, M. Flentje. Radiother. Oncol., 57 (2000), pp. $225-236$

[6] A.J.D. Scott, A.E. Nahum, J.D. Fenwick. Med. Phys., 35 (2008), pp. 4671-4684

[7] W.U. Laub, T. Wong. Med. Phys., 30 (2003), pp. 341-347

[8] I.J. Das, G.X. Ding, A. Ahnesjo. Med. Phys., 35 (2008), pp. 206-215

[9] F. Verhaegen, I.J. Das, H. Palmans. Phys. Med. Biol., 43 (1998), pp. 2755-2768

[10] D. Tromson, M. Rebisz-Pomorska, N. Tranchant, A. Isambert, F. Moignau, A. Moussier, B. Marczewska, P. Bergonzo. Diamond Relat. Mater., 19 (2010), pp. 1012-1016

[11] S. Almaviva, I. Ciancaglioni, R. Consorti, F. De Notaristefani, C. Manfredotti, M.Marinelli, E. Milani, A. Petrucci, G. Prestopino, C. Verona, G. Verona-Rinati. Nucl. Instrum. Methods Phys. Res. A Accel. Spectrom. Detect. Assoc. Equip., 608 (2009), pp. 191194

[12] I. Ciancaglioni, M. Marinelli, E. Milani, G. Prestopino, C. Verona, G. Verona-Rinati, R.Consorti, A. Petrucci, F. De Notaristefani. Med. Phys., 39 (2012), pp. 4493-4501

[13] G.T. Betzel, S.P. Lansley, F. Baluti, L. Reinisch, J. Meyer. Phys. Med., 28 (2012), pp. 144-152

[14] A. Fidanzio, L. Azario, R. Miceli, A. Russo, A. Piermattei. Med. Phys., 27 (2000), pp. 2589-2593

[15] P.W. Hoban, M. Heydarian, W.A. Beckham, A.H. Beddoe. Phys. Med. Biol., 39 (1994), pp. 1219-1229 
[16] N. Tranchant, D. Tromson, C. Descamps, A. Isambert, H. Hamrita, P. Bergonzo, M.Nesladek. Diamond Relat. Mater., 17 (2008), pp. 1297-1301

[17] Y. Garino, A. Lo Giudice, C. Manfredotti, M. Marinelli, E. Milani, A. Tucciarone, G.Verona-Rinati. Appl. Phys. Lett., 88 (2006), p. 151901

[18] F. Schirru, K. Kisielewicz, T. Nowak, B. Marczewska. J. Phys. D Appl. Phys., 43 (2010), p. 265101

[19] F. Salvat, J.M. Fernandez-Varea, J. Sempau. Penelope 2006, a Code System for Monte Carlo Simulation of Electron and Photon Transport. 92-64-02301-1, OECD, Barcelona, Spain (2006)

[20] J. Mazurier, F. Salvat, B. Chauvenet, J. Barthe. Phys. Med., 15 (1999), pp. 101-110

[21] B. Górka, B. Nilsson, J.M. Fernández-Varea, R. Svensson, A. Brahme. Phys. Med. Biol., 51 (2006), pp. 3607-3623

[22] C.D.O. Pickard, T.J. Davis, W.N. Wang, J.W. Steeds. Diamond Relat. Mater., 7 (1998), pp. $238-242$

[23] Y. Von Kaenel, J. Stiegler, J. Michler, E. Blank. J. Appl. Phys., 81 (1997), pp. 1726-1736

[24] W. Fortunato, A.J. Chiquito, J.C. Galzerani, J.R. Moro. J. Mater. Sci., 42 (2007), pp. $7331-7336$

[25] M. Pomorski, E. Berdermann, W. de Boer, A. Furgeri, C. Sander, J. Morse. Diamond Relat. Mater., 16 (2007), pp. 1066-1069

[26] H. Pernegger, S. Roe, P. Weilhammer, V. Eremin, H. Frais-Kölbl, E. Griesmayer, H.Kagan, S. Schnetzer, R. Stone, W. Trischuk, D. Twitchen, A. Whitehead. J. Appl. Phys., 97 (2005), p. 073704

[27] S. Gkoumas, A. Lohstroh, P.J. Sellin. Diamond Relat. Mater., 18 (2009), pp. 1338-1342

[28] IAEA TRS 398. Absorbed Dose Determination in External Beam Radiotherapy. An International Code of Practice for Dosimetry Based on Standards of Absorbed Dose to Water (2000) (Vienna)

[29] J.F. Fowler, F.H. Attix, W.C. Roesch (Eds.), Radiation Dosimetry, Academic, New York (1966)

[30] C.D. Angelis, S. Onori, M. Pacilio, G.A.P. Cirrone, G. Cuttone, L. Raffaele, M. Bucciolini, S. Mazzocchi. Med. Phys., 29 (2002), pp. 248-254

[31] D. Tromson, C. Descamps, N. Tranchant, P. Bergonzo, M. Nesladek, A. Isambert. J. Appl. Phys., 103 (2008), pp. 54512-54516

[32] O.A. Zeidan, J.G. Li, D.A. Low, J.F. Dempsey. Med. Phys., 31 (2004), pp. 2730-2737

[33] C. Huet, S. Dagois, S. Derreumaux, F. Trompier, C. Chenaf, I. Robbes. Radiat. Meas., 47 (2012), pp. 40-49

[34] C. Martens, C. De Wagter, W. De Neve. Phys. Med. Biol., 45 (2000), pp. 2519-2530 